\title{
The structure factor of primes
}

\author{
G. Zhang \\ Department of Chemistry, Princeton University, Princeton NJ 08544 \\ F. Martelli \\ Department of Chemistry, Princeton University, Princeton NJ 08544

\section{S. Torquato} \\ Department of Chemistry, Department of Physics, Princeton Institute for the Science \\ and Technology of Materials, and Program in Applied and Computational \\ Mathematics, Princeton University, Princeton NJ 08544 \\ torquato@electron.princeton.edu
}

\begin{abstract}
Although the prime numbers are deterministic, they can be viewed, by some measures, as pseudo-random numbers. In this article, we numerically study the pair statistics of the primes using statistical-mechanical methods, especially the structure factor $S(k)$ in an interval $M \leq p \leq M+L$ with $M$ large, and $L / M$ smaller than unity. We show that the structure factor of the prime-number configurations in such intervals exhibits well-defined Bragg-like peaks along with a small "diffuse" contribution. This indicates that the primes are appreciably more correlated and ordered than previously thought. Our numerical results definitively suggest an explicit formula for the locations and heights of the peaks. This formula predicts infinitely many peaks in any non-zero interval, similar to the behavior of quasicrystals. However, primes differ from quasicrystals in that the ratio between the location of any two predicted peaks is rational. We also show numerically that the diffuse part decays slowly as $M$ and $L$ increases. This suggests that the diffuse part vanishes in an appropriate infinite-system-size limit.
\end{abstract}

\section{Introduction}

The properties of the prime numbers have been a source of fascination since ancient times. Euclid proved that there are infinitely many primes. Given the first $n$ prime numbers $p_{1}, p_{2}, \cdots, p_{n}$, the subsequent prime can be found deterministically by sieving [1]. Nonetheless, there is no known deterministic formula that can quickly (polynomial in the number of digits in a prime) generate large numbers that are guaranteed to be prime. (So far, the largest known prime is $2^{74,207,281}-1$, which is about 22 million digits long [2].) Let $\pi(x)$ denote the prime counting function, which gives the number 
of primes less than integer $x$. According to the prime number theorem [3], the prime counting function in the large- $x$ asymptotic limit is given by

$$
\pi(x) \sim \frac{x}{\ln (x)} \quad(x \rightarrow \infty) .
$$

This means that for sufficiently large $x$, the probability that a randomly selected integer not greater than $x$ is prime is very close to $1 / \ln (x)$, which can be viewed as positiondependent number density $\rho(x)$ (number of primes up to $x$ divided by the interval $x$ ). This implies that the primes become sparser as $x$ increases and hence can be regarded as a statistically inhomogeneous set of points that are located on a subset of the odd integers.

While the prime numbers (except for 2) are a deterministic subset of the odd integers, they can be viewed, by some measures, as pseudo-random numbers. Moreover, there are quick stochastic ways to find large primes $[7,8,9,10,11]$, examples of which are based on variants of Fermat's little theorem $[7,8,9,10]$. To get a sense of how the primes can be viewed as pseudo-random numbers, let us consider the gap distribution function $P(z)$, which gives the probability distribution of the gap size between two consecutive primes, $z$. Figure 1 compares the gap probability distribution $P(z)$ for the primes to the uncorrelated lattice gas at the same number density. An "uncorrelated lattice gas" refers to a lattice-gas system where each site has a certain probability of being occupied, independent of the occupation of other sites. For an uncorrelated lattice gas at number density $\rho=N / L$ with lattice spacing 2 in the infinite-system-size limit, the gap distribution is exactly given by

$$
P(z)=f(1-f)^{z / 2-1},
$$

where $f=2 \rho$ is the probability that a site is occupied. We see that by the gap distribution, the primes cannot be clearly distinguished from an uncorrelated lattice gas. Indeed, probabilistic methods to treat the primes have yielded fruitful insights about them $[4,5,6]$. For example, based on the assumption that the primes behave like a Poisson process (uncorrelated lattice gas), Cramér (1920) conjectured that [4] for large $x$

$$
g(x) \geq c \ln ^{2}(x)
$$

where $g(x)$ denotes the largest prime gap within an interval $[x, 2 x]$.

On the other hand, it is known that primes contain unusual patterns. Chebyshev observed in 1853 that primes congruent to 3 modulo 4 seem to predominate over those congruent to 1 [12]. Assuming a generalized Riemann hypothesis, Rubinstein and Sarnak [13] exactly characterized this phenomenon and more general related results. A computational study on the Goldbach conjecture demonstrates a connection based on a modulo 3 geometry between the set of even integers and the set of primes [14]. In 1934, Vinogradov proved that every sufficiently large odd integer is the sum of three primes [15]. This method has been extended to cover many other types of patterns $[16,17,18,19]$. Recently it has been shown that there are infinitely many 


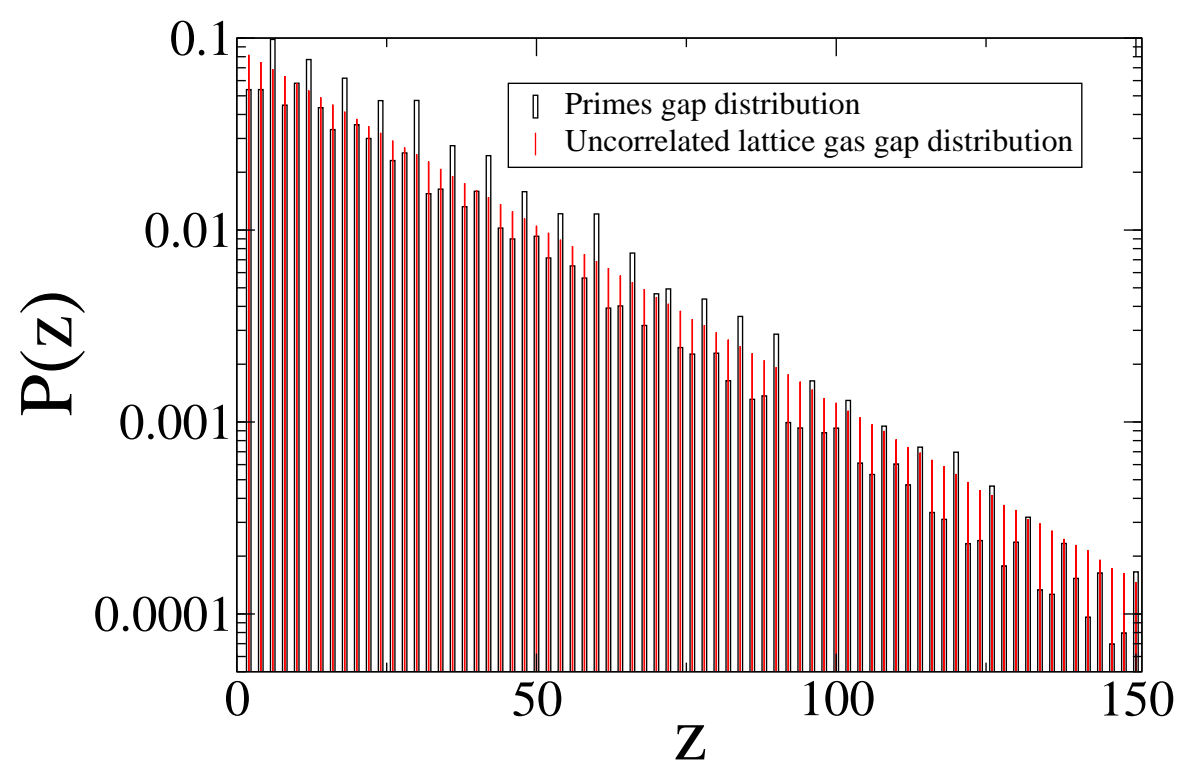

Figure 1. Comparison of the gap distribution for the primes and the uncorrelated lattice gas with the same cardinality (occupation number) as the set of primes. The primes are taken to lie on an integer lattice with a spacing of 2, i.e., a subset of the odd positive integers. We consider $N$ primes in interval $[M, M+L](M$ large and $M \gg L)$. Here $N=10^{7}, L=244651478$ with $M=42151671493$, the $1,800,000,000$ th prime number.

pairs of primes with some finite gap [20] and that primes ending in 1 are less likely to be followed by another prime ending in 1 [21]. Numerical evidence of regularities in the distribution of gaps between primes when these are divided into congruence families have also been reported $[22,23,24]$, along with the observation of period-three oscillations in the distribution of increments of the distances between consecutive primes numbers [25].

The present paper is motivated by certain unusual properties of the Riemann zeta function $\zeta(s)$, which is a function of a complex variable $s$ that is intimately related to the primes. The zeta function has many different representations, one of which is the series formula

$$
\zeta(s)=\sum_{n=1}^{\infty} \frac{1}{n^{s}},
$$

which only converges for $\operatorname{Re}(s)>1$. However, $\zeta(s)$ has a unique analytic continuation to the entire complex plane, excluding the simple pole at $s=1$. According to the Riemann hypothesis, the nontrivial zeros of the zeta function lie along the critical line $s=1 / 2+i t$ with $t \in \mathbb{R}$ in the complex plane. The nontrivial zeros tend to get denser the higher on the critical line. When the spacings of the zeros are appropriately normalized so that they can be treated as a homogeneous point process at unity density, the resulting pair correlation function takes on the simple form $1-\sin ^{2}(\pi r) /(\pi r)^{2}[6]$. The corresponding structure factor $S(k)$ (essentially the Fourier transform of $\left.g_{2}(r)\right)$ tends to zero linearly in the wavenumber $k$ as $k$ tends to zero but is unity for sufficiently large $k$. This implies that the normalized Riemann 
zeros possess a remarkable type of correlated disorder at large length scales known as hyperuniformity [26]. A hyperuniform many-particle system is one in which the structure factor approaches zero in the infinite-wavelength limit [27]. In such systems, density fluctuations are anomalously suppressed at very large length scales, a "hidden" order that imposes strong global structural constraints. All structurally perfect crystals and quasicrystals are hyperuniform, but typical disordered many-particle systems, including gases, liquids, and glasses, are not. Disordered hyperuniform many-particle systems are exotic states of amorphous matter that have attracted considerable recent attention $[28,29,30,31,32,33,34,35,36,37,38,39,40,41,42,43,44,45]$. The zeta function is directly related to the primes via the following Euler product formula:

$$
\zeta(s)=\left[\prod_{n=1}^{\infty}\left[1-1 / p_{n}^{s}\right]\right]^{-1},
$$

which leads to a variety of explicit formulas that link the primes on the one hand to the zeros of the zeta function on the other hand $[46,47,48]$. Thus, one can in principle deduce information about primes from information about zeros of the zeta function. Accordingly, one might expect the primes to encode hyperuniform correlations that are seen in the Riemann zeros.

In this article, we numerically study the pair statistics of configurations of the primes, especially the structure factor $S(k)$ in an interval $M \leq p \leq M+L$ with $M$ large, and $L / M$ smaller than unity. As we will detail in Sec. 2.3, this choice of intervals allow us to obtain prime configurations with virtually constant density from one end of the interval to the other. We show in Sec. 3 that the structure factor exhibits welldefined Bragg-like peaks along with a small "diffuse" contribution. This indicates that the primes are appreciably more correlated than anyone has previously conceived. Our numerical results definitively suggest an explicit formula for the locations and heights of the peaks. This formula predicts infinitely many peaks in any non-zero interval. Although such a behavior is similar to that of quasicrystals [51, 52, 53], primes differ from the latter in that the ratio between the locations of any two predicted peaks is rational. We also show numerically that the diffuse part decays slowly as $M$ and $L$ increases. In a subsequent paper [55], we prove this to be true and investigate its consequences. This might indicate that the diffuse part vanishes in an appropriate infinite-system-size limit. We note that the structure factor has been used to study other number-theoretic systems [7, 49, 26, 50].

The rest of the paper is organized as follows: In Sec. 2, we present relevant definitions and describe the simulation procedure. In Sec. 3, we present results for the pair statistics of the primes in certain intervals in both direct and reciprocal spaces. In Sec. 4, we make concluding remarks. 


\section{Definitions and Simulation Procedure}

We will study the pair correlation function as well as the structure factor of primenumber configurations. Similar to Ref. [54], we treat the primes in some interval $[M, M+L]$ to be a special lattice-gas model: the primes are "occupied" sites on a integer lattice of spacing 2 that contains all of the positive odd integers and the unoccupied sites are the odd composite integers. As detailed below, we consider the positive parameter $L / M$ generally to be smaller than unity to ensure homogeneity. Within this interval, let $N_{s}$ and $N$ be the total number of sites and the total number of primes, respectively. In practice, we consider the half-open interval $[M, M+L)$ because it provides a simple relation between $L$ and $N_{s}$, namely, $L=2 N_{s}$. For all cases considered in this paper, $M \geq 3$. Figure 2 illustrates an example of a prime configuration. The rest of this section details the mathematical tools that we use to treat such systems.

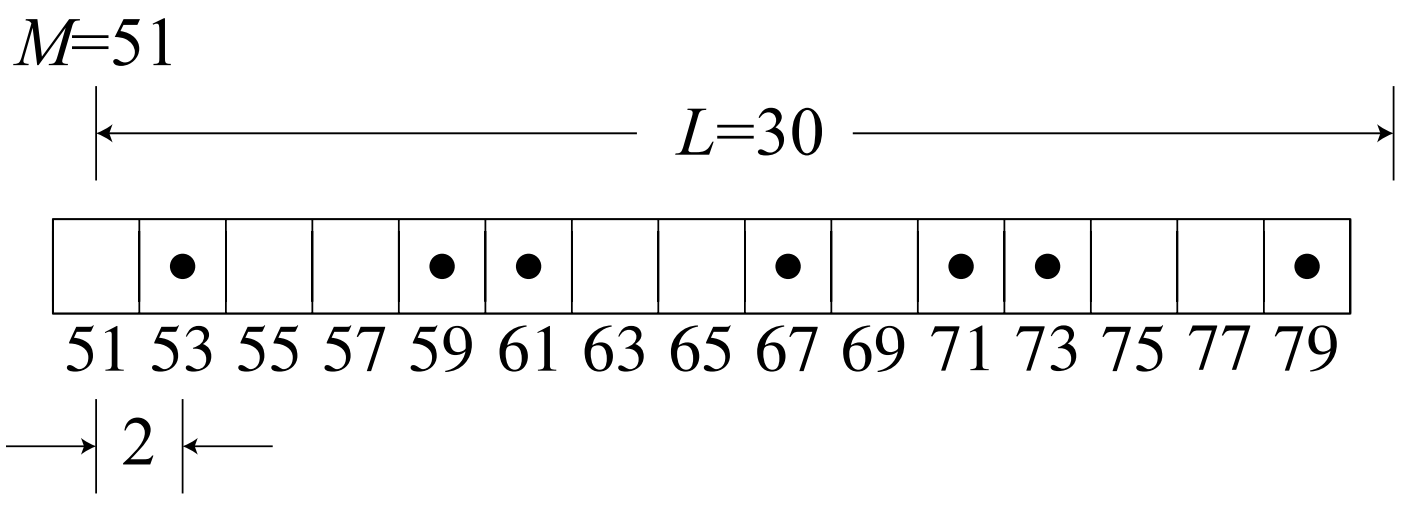

Figure 2. Schematic plot of a prime-number configuration with $M=51$ and $L=30$. Since we always use $M \geq 3$, any prime number in the interval $[M, M+L)$ is odd. Therefore, a prime-number configuration is a lattice gas with lattice spacing 2 in which the primes are the "occupied" sites and the composites are "unoccupied" sites.

\subsection{Discrete Fourier Transform}

For a function $f(r)$ defined on an integer lattice with spacing $a$ that is contained within a periodic box of length $L$, one may define its Fourier transform as follows:

$$
\tilde{f}(k)=\sum_{r=0, a, 2 a, \cdots, L-a} f(r) \exp (i k r),
$$

where the parameter $k$ is an integer multiple of $2 \pi / L$. The inverse transform is given by

$$
f(r)=\frac{1}{N_{s}} \sum_{j=0}^{N_{s}-1} \tilde{f}\left(\frac{2 \pi j}{L}\right) \exp \left(-i \frac{2 \pi j}{L} r\right),
$$

where $N_{s}=L / a$ is the number of sites. 


\subsection{Pair Statistics and their basis properties}

We define $\eta(r)$ as the indicator function such that $\eta(r)=1$ if the site at $r$ is occupied, and $\eta(r)=0$ otherwise. Let $\tilde{\eta}(r)$ be its Fourier transform. We define occupation fraction to be $f=<\eta(r)>$, where $<>$ denotes an average over all $r$. We define the structure factor as

$$
S(k)=|\tilde{\eta}(k)|^{2} / N-N \delta_{k, 0} .
$$

Define the pair correlation function $g_{2}(r)$ as

$$
g_{2}(r)=\frac{1}{N f} \sum_{n=0, a, 2 a, \cdots, L-a} \eta(n) \eta(n+r)-\frac{\delta_{r, 0}}{f} .
$$

By definition, $g_{2}(0)=0$. For $r \neq 0, g_{2}(r)$ can be interpreted as the probability that the site at $p+r$ is occupied given that the site at $p$ is occupied divided by $f$.

The structure factor and the pair correlation function are related as follows:

$$
\begin{aligned}
N \delta_{k, 0}+S(k) & =|\tilde{\eta}(k)|^{2} / N \\
& =\frac{1}{N} \sum_{m=0, a, 2 a, \cdots, L-a} \eta(m) \exp (i k m) \sum_{n=0, a, 2 a, \cdots, L-a} \eta(n) \exp (-i k n) \\
& =\frac{1}{N} \sum_{m=0, a, 2 a, \cdots, L-a} \eta(m) \eta(n) \exp [i k(m-n)] \\
& =\frac{1}{N} \sum_{r=0, a, 2 a, \cdots, L-a} \sum_{n=0, a, 2 a, \cdots, L-a} \eta(n+r) \eta(n) \exp (i k r) \\
& =\frac{1}{N} \sum_{n=0, a, 2 a, \cdots, L-a} \eta(n+0) \eta(n) \exp (i k 0) \\
& +\frac{1}{N} \sum_{r=a, 2 a, \cdots, L-a} \sum_{n=0, a, 2 a, \cdots, L-a} \eta(n+r) \eta(n) \exp (i k r) \\
& =\frac{N}{N}+\frac{1}{N} \sum_{r=a, 2 a, \cdots, L-a} N f g_{2}(r) \exp (i k r) \\
& =1+f \sum_{r=a, 2 a, \cdots, L-a} g_{2}(r) \exp (i k r) \\
& =1+f \sum_{r=0, a, \cdots, L-a} g_{2}(r) \exp (i k r)
\end{aligned}
$$

This equation enables us to obtain a sum rule for both $g_{2}(r)$ and $S(k)$. For $g_{2}(r)$, plugging $k=0$ into Eq. (10) yields

$$
\frac{N-1}{f}=\sum_{r=a, 2 a, \cdots, L-a} g_{2}(r) .
$$

The sum rule for $S(k)$ is easily found by invoking the inverse Fourier transform equation:

$$
g_{2}(r)=\frac{1}{N_{s} f} \sum_{j=0}^{N_{s}-1}\left[S\left(\frac{2 \pi j}{L}\right)+N \delta_{k, 0}-1\right] \exp \left(-i \frac{2 \pi j}{L} r\right) .
$$


At $r=0$, this relation becomes

$$
\begin{aligned}
0 & =\sum_{j=0}^{N_{s}-1}\left[S\left(\frac{2 \pi j}{L}\right)+N \delta_{k, 0}-1\right] \\
& =\sum_{j=1}^{N_{s}-1} S\left(\frac{2 \pi j}{L}\right)+N-N_{s},
\end{aligned}
$$

and hence the sum rule for the structure factor is given by

$$
\sum_{j=1}^{N_{s}-1} S\left(\frac{2 \pi j}{L}\right)=N_{s}-N
$$

For the primes, $L=2 N_{s}$, and hence the sum rule is specifically

$$
\sum_{j=1}^{N_{s}-1} S\left(\frac{\pi j}{N_{s}}\right)=N_{s}-N .
$$

The fact that all primes greater than 3 are odd integers lead to a few important properties of $S(k)$. First, $N \delta_{k, 0}+S(k)$ is a periodic function of period $\pi$, since

$$
\begin{aligned}
N \delta_{k+\pi, 0}+S(k+\pi) & =\frac{\left|\sum_{j=1}^{N} \exp \left[-i(k+\pi)\left(p_{j}-M\right)\right]\right|^{2}}{N} \\
& =\frac{\left|\sum_{j=1}^{N} \exp \left[-i k\left(p_{j}-M\right)\right] \exp \left(-i \pi\left(p_{j}-M\right)\right]\right|^{2}}{N} \\
& =\frac{\left|-\exp (i \pi M) \sum_{j=1}^{N} \exp \left[-i k\left(p_{j}-M\right)\right]\right|^{2}}{N} \\
& =\frac{\left|\sum_{j=1}^{N} \exp \left[-i k\left(p_{j}-M\right)\right]\right|^{2}}{N} \\
& =N \delta_{k, 0}+S(k),
\end{aligned}
$$

Second, from Eqs. (6) and (8), one can see that when $k=m \pi$, where $m$ is any nonzero integer, $S(k)=N$ achieves the global maximum of this function. The function $S(k)$ therefore displays strong peaks at such $k$ values. Third, from Eqs. (6) and (8), one can see the function $S(k)$ has reflection symmetry $S(k)=S(-k)$. This reflection symmetry, combined with the periodicity [Eq. (16)], implies another reflection symmetry, $S(\pi / 2+k)=S(\pi / 2-k)$. With these properties in mind, we only need to study $S(k)$ in the range $0<k \leq \pi$ in this paper.

\subsection{Simulation Procedure}

In statistical mechanics, the study of $S(k)$ often focuses on statistically homogeneous systems. However, the prime numbers are not homogeneous. Instead, in the vicinity of $x$, the density of prime numbers scales as $1 / \ln (x)$. To overcome this difference, we focus 
on large $M$ values and let $L / M$ be a constant less than unity $\ddagger$. This implies that the "local" density from the beginning to the end of the interval $[M, M+L)$ is virtually a constant. For example, we will study a system of $M=10^{10}$ and $L=10^{7}$. As $x$ changes from $M$ to $M+L, 1 / \ln (x)$ only changes from $0.043429 \ldots$ to $0.043427 \ldots$.

We minimize the problem of inhomogeneity by requiring sufficiently large $M$. Instead, one might think an even better solution to this problem is to rescale the configuration such that it is homogeneous. The natural scaling is to replace each prime number $p$ with $p / \ln (p)$. However, it turns out that after performing such rescaling, the structure factor appears to be completely noisy with no obvious peaks with heights comparable to N; see Fig. $3 \S$. This is to be contrasted with our findings reported in the rest of the paper, in which we choose to study the primes in the interval $[M, M+L)$ with $M$ large, and $L / M$ smaller than unity.

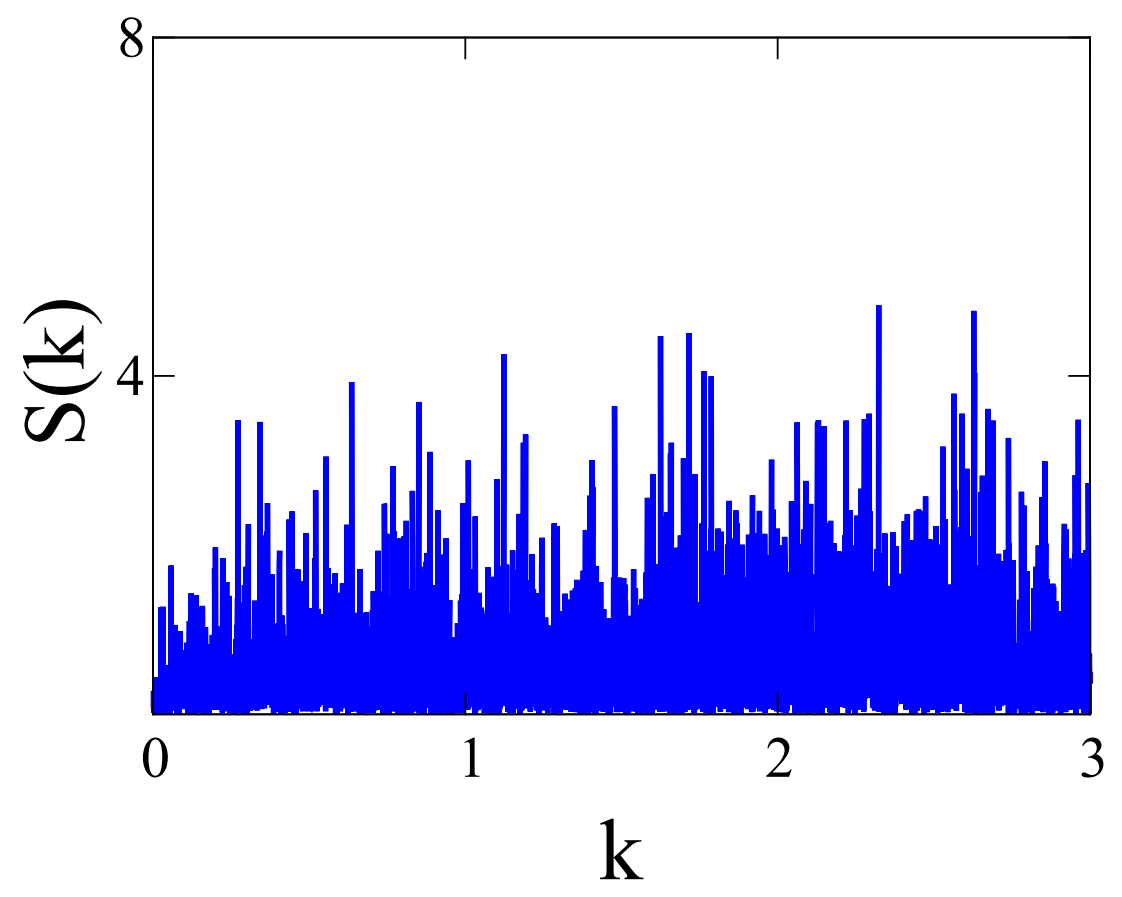

Figure 3. Structure factor $S(k)$ associated with $p / \ln (p)$ for all prime number $p$ 's in the interval $\left[3,3+10^{5}\right)$.

We obtain a list of prime numbers from Ref. [56], and calculate $S(k)$ of prime numbers and uncorrelated lattice gases with the fast Fourier transform (FFT) algorithm using the kissFFT software [57]. This algorithm has the advantage of not only being

$\ddagger$ Strictly speaking, the requirement that $L / M<1$ is not important. Densities from one end to the other would be nearly constant in the $M \rightarrow \infty$ limit even if $L / M=\beta>1$. However, for computationally practical values of $M$, a much smaller $L$ is desired to accurately ascertain constant density.

$\S$ Notice that in this interval, primes possess a sharp density gradient, and the $p \rightarrow p / \ln (p)$ rescaling is strongly non-linear. If we had chosen an interval with a negligible density gradient, then the $p \rightarrow p / \ln (p)$ rescaling would be almost linear, and $S(k)$ would essentially be a simple rescaling of the ones reported below (Fig. 5). 


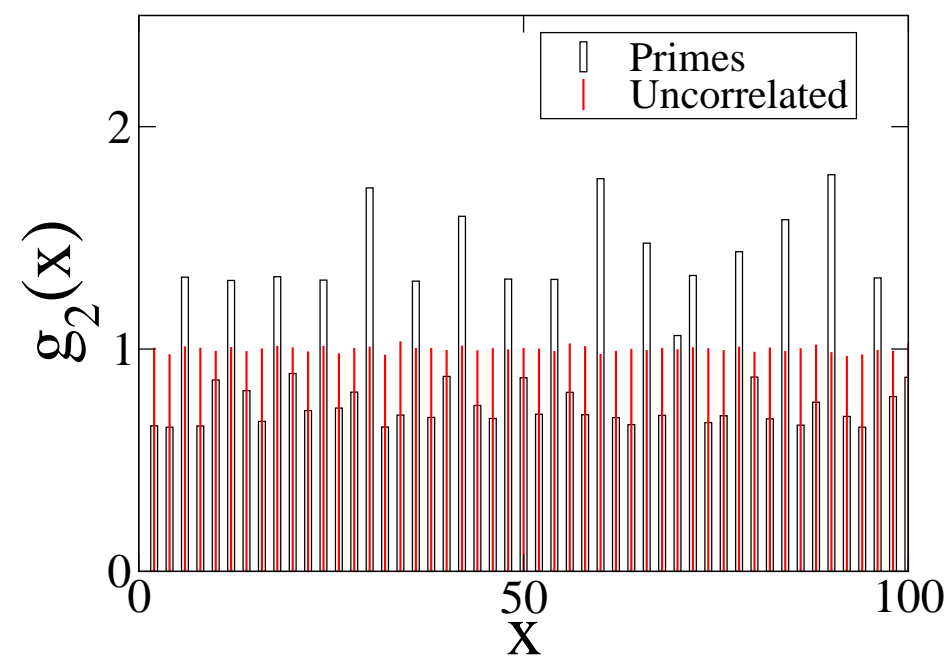

Figure 4. Pair correlation function $g_{2}(x)$, as defined in Eq. (9), for a prime number configuration of $M=10^{10}+1, L=10^{6}$, and $N=43427$, compared with $g_{2}(x)$ of a uncorrelated lattice gas configuration of the same $L$ and $N$.

"fast" $\|$, but also being accurate, as the upper bound on the relative error scales as $\epsilon \log (L)$, where $\epsilon$ is the machine floating-point relative precision. We use double-precision numbers to further minimize $\epsilon$.

More precisely, FFT allows one to efficiently calculate

$$
X(q)=\sum_{n=0}^{T-1} x_{n} \exp (-2 \pi i q n / T)
$$

for arbitrary $x_{0}, x_{1}, \cdots, x_{T-1}$. Here, we simply let $T=L / 2$, and let $x_{j}=1$ if $M+2 j$ is a prime number and $x_{j}=0$ if $M+2 j$ is composite. The structure factor is then calculated from:

$$
S(2 \pi q / L)=|X(q)|^{2} / N
$$

\section{Results for the Pair Statistics of the Prime Numbers}

The pair correlation function as defined in Eq. (9), $g_{2}(x)$, of prime numbers is presented in Fig. 4 and compared with $g_{2}(x)$ of uncorrelated lattice gases. This quantity for uncorrelated lattice gas is simply

$$
g_{2}(x)=\frac{N-1}{f\left(N_{s}-1\right)}
$$

for any $x \neq 0$. This is because after one site is occupied, out of the remaining $N_{s}-1$ sites, exactly $N-1$ sites are occupied. We see that by this measure, the prime numbers appear to be distinctly different from uncorrelated lattice gases. We see that $g_{2}(x)$ for \| The time complexity of calculating $S(k)$ for all $k$ 's using FFT algorithm scales as $L \log (L)$, while the time complexity of doing so using Eq. (10) scales as $L N$. 
primes is higher than $g_{2}(x)$ of the uncorrelated lattice gas if and only if $x$ is divisible by 3. However, we will see that the difference in pair statistics is much more obvious when we study $S(k)$ below.

We present and study numerically calculated structure factors of prime numbers for various $M$ 's and $L$ 's in this section. At first, let us examine $S(k)$ for $M=10^{6}+1$ and $L=5000$, which is presented in Fig. 5. At a larger scale, $S(k)$ appears to consist of many well-defined Bragg-like peaks of various heights, with the highest peak occurring at $k=\pi$ (left panel). As we zoom in, it becomes evident that besides those peaks, $S(k)$ also has a random, noisy contribution that is often below 1 (right panel). We will call the latter contribution the "diffuse part" in the rest of the paper. Figure 5 also includes $S(k)$ for uncorrelated lattice gases, which consists of a diffuse part and a single peak at the trivial value of $k=\pi$. Away from the peak, $S(k)$ for uncorrelated lattice gases fluctuates around an average value of $\frac{N_{s}-N}{N_{s}-1}$ [this particular average value is required by the sum rule, Eq. (14)]. A major conclusion is that the structure factor of the primes is characterized by a substantial amount of order across length scales, relative to the uncorrelated lattice gas, as evidenced by the appearance of many Bragglike peaks. At this stage, it seems that the existence of the diffuse part makes primes non-hyperuniform. However, we will show in Sec. 3.2 that the diffuse part decreases as $L$ increases, and suggest that it vanishes in the infinite-system-size limit.
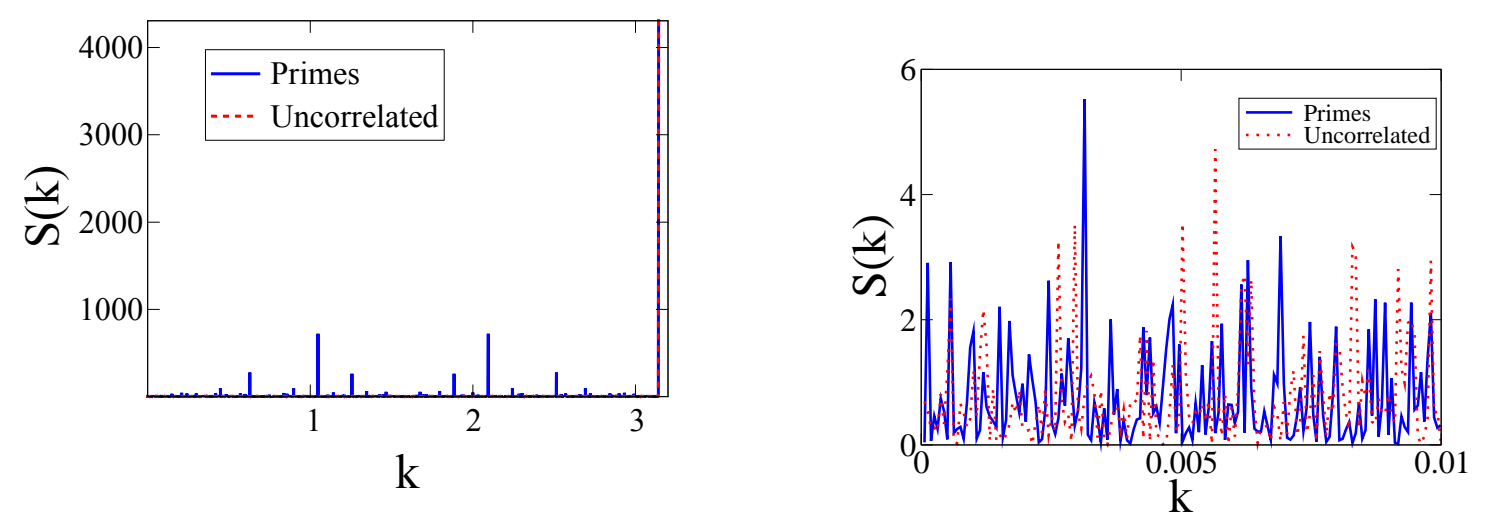

Figure 5. Left: $S(k)$ for prime numbers as a function of $k$ (in units of the integer lattice spacing) for $M=10^{10}+1$ and $L=10^{5}$ contains many well-defined Bragg-like peaks of various heights, creating a type of self-similarity. Right: A zoomed-in view revealing the existence of a small, noisy "diffuse part" besides the peaks. We also plot $S(k)$ for uncorrelated lattice gases for comparison. As we have discussed in Sec. 2, we only show $S(k)$ in the range $0<k \leq \pi$, and therefore omit the peak at $k=0$.

At this stage, the distinction between the peaks and the "diffuse part" is somewhat unclear. Since $S(k)$ contains peaks of various heights, is it possible that the diffuse part is actually made of many smaller peaks? We can only answer this question after we study the peaks and the diffuse part more deeply later in this section. 


\subsection{Peaks}

We move on to study the peaks. From Fig. 5, one sees that the highest peak is at $k=\pi$, which is trivially caused by the periodicity of the underlying lattice. The next highest two peaks are at $k=\pi / 3$ and $k=2 \pi / 3 \boldsymbol{q}$. Even lower peaks occur at $k=\pi / 5,2 \pi / 5$, $3 \pi / 5$, and $4 \pi / 5$. Still lower peaks occur at $k=\pi / 7,2 \pi / 7,3 \pi / 7,4 \pi / 7, k=5 \pi / 7$, and $6 \pi / 7$. Examining $S(k)$ of a much larger system $\left(M=10^{10}+1\right.$ and $\left.L=10^{7}\right)$ revealed that there are even more peaks with locations that obey the formula $k=m \pi / n$, where $m$ is any integer coprime with $n$ and $n$ is any square-free odd integer and hence has a distinct prime factorization, i.e., $n=\prod_{j=1}^{J} p_{j}$, where $J$ is a positive integer, and $p_{1}, p_{2}$, $\cdots, p_{J}$ are non-repeating prime numbers larger than 2. If $n$ is even or is not square-free, then we observe no peak at $k=m \pi / n$. We verified the existence of such peaks for $n$ up to 300. As $n$ increases beyond 300, however, the peaks become too weak to be distinguishable from the diffuse part.

Having an analytical formula of the peak locations, we move on to study the peak heights. As we have shown earlier, the height of the peak at $k=\pi$ is simply $N$. What can we say about the heights of the other peaks? In Fig. 6 we present computed peak heights at $k=\pi / 3$ and $k=\pi / 5$ for $M=10^{10}+1$ and various $L$ 's. We see that as $L$ grows, the heights of the peaks at $k=\pi / 3$ and $k=\pi / 5$ also grow and remain roughly proportional to $N$. Looking at the inset, we see that both $S(\pi / 3)$ and $S(\pi / 5)$ oscillates periodically as $L$ increases: $S(\pi / 3)$ attains a maximum when $L$ is divisible by 3 , and $S(\pi / 5)$ attains a maximum when $L$ is divisible by 5 . Examining the heights of other peaks, we find that the height of a peak at $k=m \pi / n$ is indeed highest when $L$ is divisible by $n$.

Since the divisibility of $L$ with $n$ affects the peak heights and hence will introduce unintended errors if not chosen properly, we desire an $L$ that is divisible by as many prime numbers as possible. We therefore chose $L=2 \times 3 \times 5 \times 7 \times 11 \times 13 \times 17 \times 19=$ 9699690 and recomputed the heights of several peaks. The results are summarized in Table 1 . We find that when $L$ is divisible by $n$, the height of the peak at $k=m \pi / n$ is very close to $N \prod_{j=1}^{J}\left(p_{j}-1\right)^{-2}$, where $p_{j}$ are the distinct prime factors of $n$.

Do these numerically generated peaks have finite or infinitesimal width? To answer this question, we present a close view of the peak at $k=\pi / 3$ for three different $L$ 's in Fig. 7. It turns out that, if $L$ is divisible by $n$, the peak at $k=m \pi / n$ has infinitesimal width, in the sense that $S(k)$ at one $k$ value attains the local maximum and $S(k)$ at all adjacent $k$ values are as low as the typical diffuse part. However, if $L$ is not divisible by $n$, then the peak has a finite width, as $S(k)$ of all $k$ values very close to $m \pi / n$ rises and become much higher than the typical diffuse part. In Fig. 7 one can also see that when peak widths are finite, a lower $L$ results in a more broadly spread peak. Therefore, all

I It should be noted that since $k$ has to be integer multiples of $2 \pi / L$, for $L=10^{5}, k=\pi / 3$ and $k=2 \pi / 3$ cannot be chosen. The actually observed peaks occur at the closest allowed $k$ points instead. 


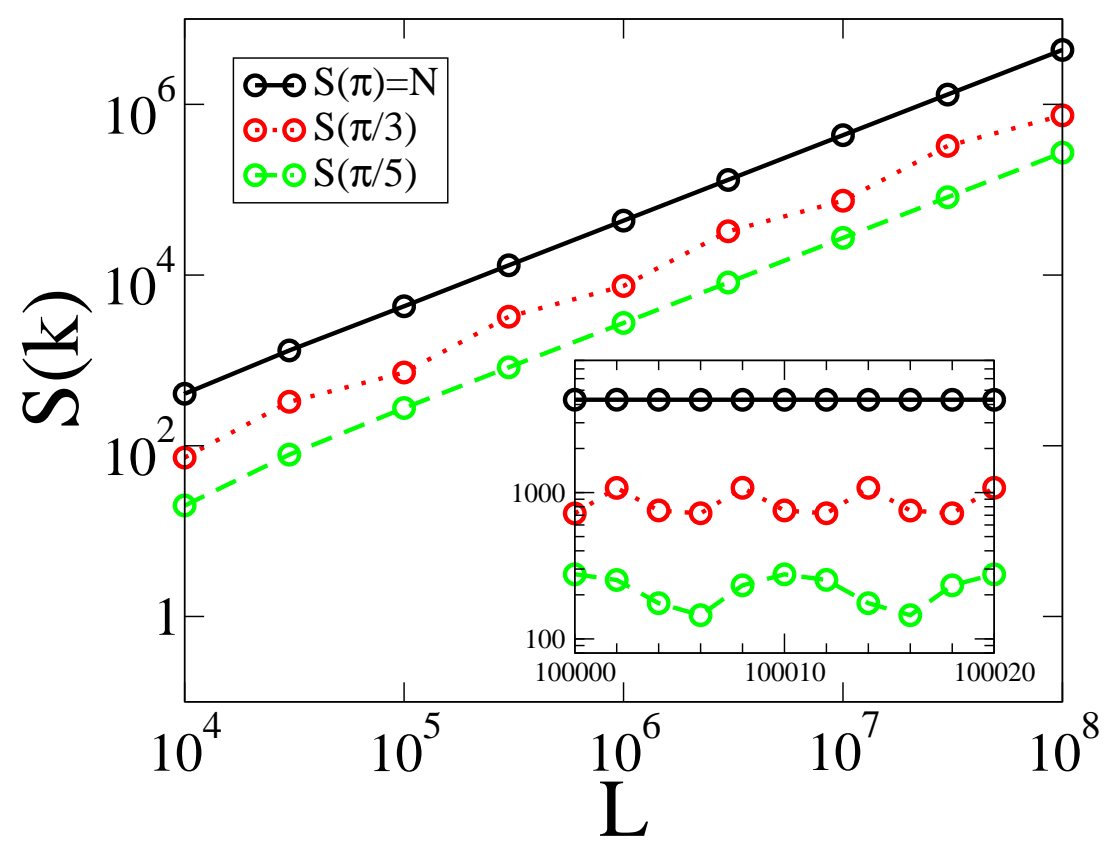

Figure 6. The structure factor $S(k)$ at $k=\pi, k=\pi / 3$, and $k=\pi / 5$, as a function of $L$ at $M=10^{10}+1$. The inset presents more data for $10^{5} \leq L \leq 10^{5}+20$.

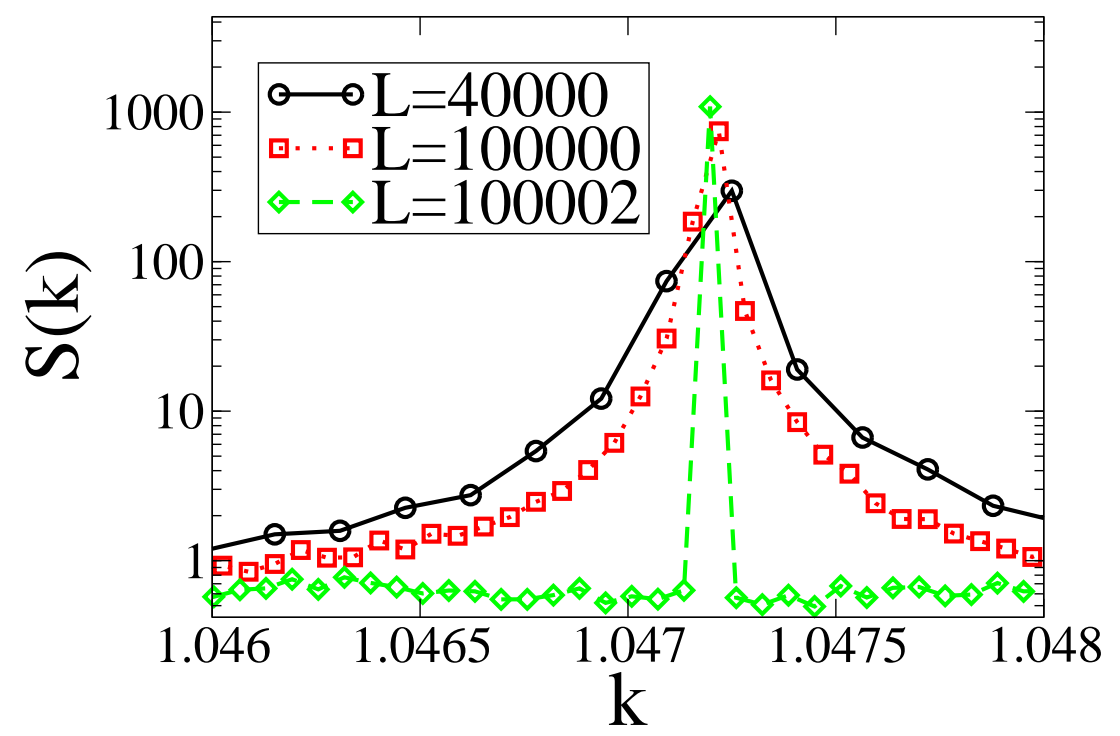

Figure 7. $S(k)$ near $k=\pi / 3$ for three different $L$ 's. Each curve is averaged over 100 prime-number configurations, with the $j$ th configuration consists of all prime numbers in the range $\left[10^{10}+(j-1) L+1,10^{10}+j L+1\right)$.

of the peaks may have infinitesimal width in the infinite- $L$ limit. However, in a finite$L$ simulation, choosing an $L$ that is divisible by as many prime numbers as possible provides a better estimate of $S(k)$ in the infinite- $L$ limit. As $n$ increases, one finds an increasing number of lower peaks, resulting in a statistical self-similarity. 
Table 1. Peak heights at several different $n$ and $m$ 's for $M=2.5 \times 10^{8}+1$ and $L=9699690$ and comparison with the predicted height from the analytical formula.

\begin{tabular}{|c|c|c|c|}
\hline$n$ & $m$ & $S(m \pi / n) / N$ & Postulated analytical formula \\
\hline 3 & 1 & 0.2500000003 & $(3-1)^{-2}=0.25$ \\
\hline 5 & $\frac{1}{2}$ & 0.06268293536 & $(5-1)^{-2}=0.0625$ \\
\hline \multirow{3}{*}{7} & 1 & 0.02764696627 & \multirow{3}{*}{$(7-1)^{-2}=0.02777 \cdots$} \\
\hline & 2 & 0.02783423055 & \\
\hline & 3 & 0.02785282486 & \\
\hline \multirow{3}{*}{$15=3 \times 5$} & 1 & 0.01564115190 & \multirow{3}{*}[(3-1)(5-1)]{$^{-2}=0.015625$} \\
\hline & $\frac{2}{4}$ & $\begin{array}{l}0.01583266309 \\
0.01551814312\end{array}$ & \\
\hline & 7 & $\frac{0.01031014012}{0.01550964066}$ & \\
\hline \multirow{24}{*}{$105=3 \times 5 \times 7$} & 1 & 0.0004096963803 & \multirow{24}{*}[(3-1)(5-1)(7-1)]{$^{-2}=0.00043402777 \cdots$} \\
\hline & 2 & 0.0004418025682 & \\
\hline & 4 & 0.0004305924622 & \\
\hline & 8 & 0.0003879974866 & \\
\hline & II & 0.0004203223484 & \\
\hline & 13 & 0.0004411107279 & \\
\hline & 16 & 0.0004191249498 & \\
\hline & 17 & 0.0003893716268 & \\
\hline & 19 & 0.0004388128207 & \\
\hline & 22 & 0.0004193036024 & \\
\hline & 23 & 0.0004375599695 & \\
\hline & 26 & 0.0004203613535 & \\
\hline & 29 & 0.0004418187004 & \\
\hline & 31 & 0.0004457650582 & \\
\hline & 32 & 0.0004237635619 & \\
\hline & 34 & 0.0004500979160 & \\
\hline & 37 & 0.0004466597486 & \\
\hline & 38 & 0.0004663304920 & \\
\hline & 41 & 0.0004255673779 & \\
\hline & 43 & 0.0004845933410 & \\
\hline & 44 & 0.0004679589962 & \\
\hline & 46 & 0.0004572985410 & \\
\hline & 47 & 0.0004095637658 & \\
\hline & 52 & 0.0004521962772 & \\
\hline
\end{tabular}

\subsection{Diffuse Part}

The above analysis suggest that the structure factor of the primes possesses infinitely many Dirac-delta-function peaks of various heights in the infinite-system-size limit. Therefore, one might naturally ask, could the random, noisy "diffuse part" be simply a superposition of many small peaks? The answer is no. In Fig. 8 we present $S(k)$ of two different $L$ 's in the range $0.2 \leq k \leq 0.24$. For $L=5000, S(k)$ in this range appeared completely random and noisy, matching our definition of the diffuse part. To see if this diffuse part is actually a superposition of many small peaks, we compare it to the structure factor for $L=500000$. The larger $L$ allows more $k$ points to be chosen, and therefore improves the $k$ resolution, and reveals some peaks in this $k$ range. We see that although $S(k)$ of the smaller $L$ appeared to be entirely random, the maximum at $k \approx 0.21$ corresponds to a strong peak of the larger system, and is therefore actually a peak. However, other maxima for the smaller system do not correspond to peaks for the larger system, and can only be explained by assuming the existence of a noisy 


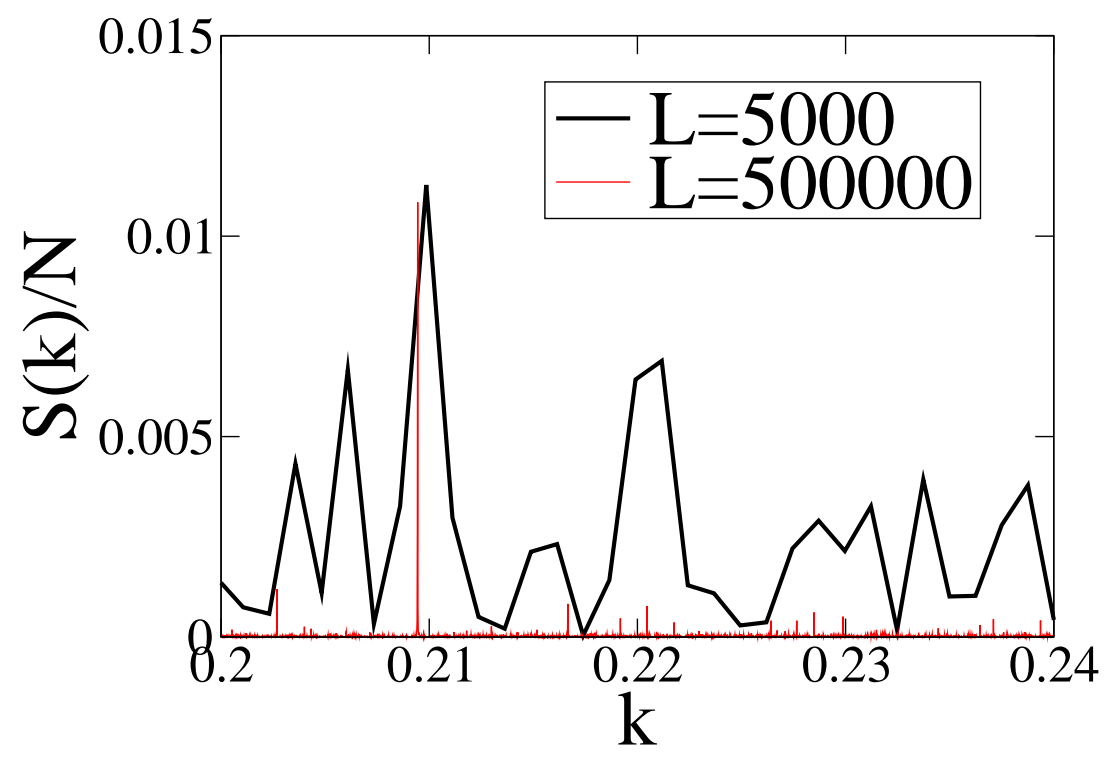

Figure 8. The structure factor, $S(k)$, normalized by $N$, for two different $L$ 's and $M=10^{6}+1$.

contribution to $S(k)$, which we call the "diffuse part." To summarize, in Fig. 8 we show that for finite systems, there is clearly a noisy contribution to $S(k)$ other than the peak contribution, even though distinguishing these two components of $S(k)$ can be difficult without consulting the analytical formula for the peaks.

The diffuse part contributes to not only $k$ points where there are no peaks, but also to $k$ points where there are peaks. In Fig. 9, we compare numerical peak heights, averaged over all allowed $m$ for a particular $n$, with the analytic peak formula described in Sec. 3.1. It turns out that the numerical average is always slightly higher than that predicted by this formula, and their difference is of the same order of magnitude as the diffuse part. Thus, $S(k)$ at predicted peak locations is actually the sum of the peak and diffuse contributions.

We can quantify the diffuse part using the median of $S(k)$ for all possible choices of $k$ in the range $0<k \leq \pi$. We present these medians in Fig. 10. As $L$ increases, the median of $S(k)$ generally decreases. However, with our current data, it is unclear if the median of $S(k)$ approaches zero in the $L \rightarrow \infty$ limit.

\section{Conclusions}

In summary, we have numerically shown that the structure factor of prime numbers in the interval $M \leq p<M+L$ with large $M$ and $L / M<1$ exhibit well-defined Bragglike peaks, alongside a very small diffuse part, which slowly decays as the system size increases. In contrast, the peaks persist as the system size increases. Therefore, we have numerically shown in this paper that the primes are characterized by a substantial amount of order, especially relative to an uncorrelated lattice gas that does not have any such peaks. We also show that peaks at $k=m \pi / n$ are sharper when $L$ is divisible 
$\mathrm{M}=10^{10}+1, \mathrm{~L}=9699690$

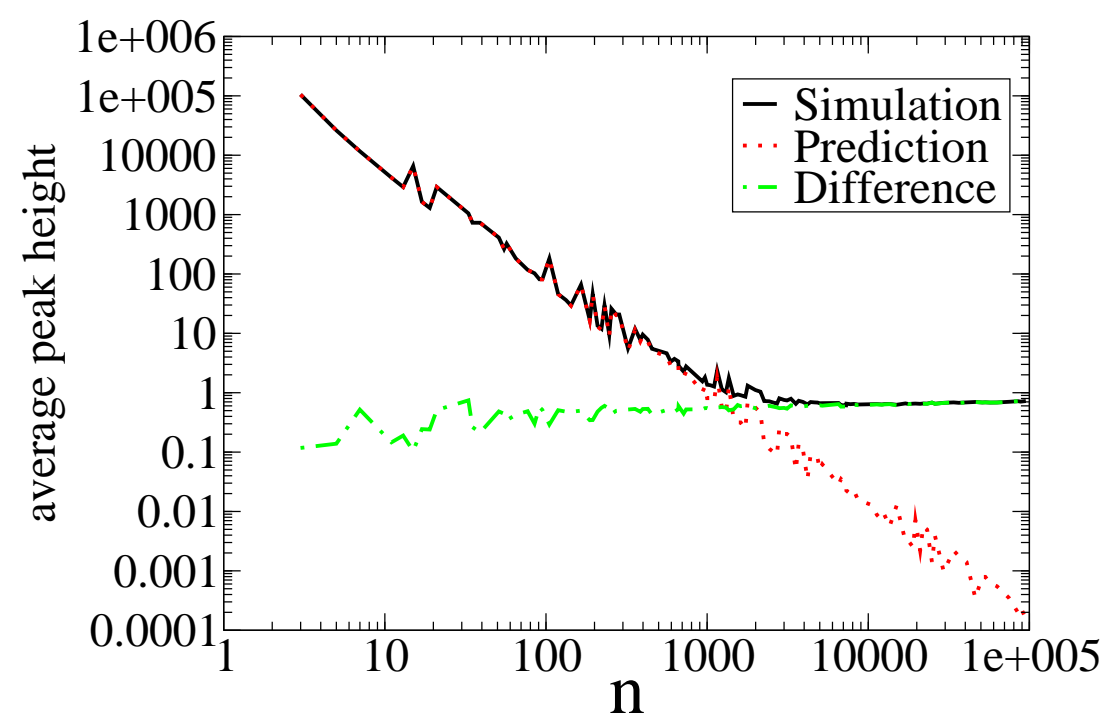

Figure 9. Average peak height of all peaks of a given $n$, the predicted peak heights, and their difference for all $n<10^{5}$ that are odd, square-free, and divide $L$ evenly. Here $M=10^{10}+1$ and $L=9699690$. For each $n$, we find all $m$ 's that are coprime with $n$, and average the heights of peaks at $m \pi / n$. The average turns out to be always greater than the prediction, $N \prod_{j=1}^{J}\left(p_{j}-1\right)^{-2}$. Their difference is between 0.1 and 1 , which is of the same order of magnitude as the diffuse part.

by $n$. Our numerical results definitively suggested an explicit formula for the peak locations and heights, which predicts dense Bragg peaks, as occurs in quasicrystals (e.g., Fibonacci chain [51] as well as other one-dimensional examples [53]), but unlike the latter, the peak locations occur at rational multiples of $\pi$. In an upcoming paper [55], we will show that the primes in the intervals studied here are similar to "limit periodic" systems [50] but still distinct from them. Limit-periodic systems are unions of an infinite number of periodic systems with rational periods, and possess dense Bragg peaks with rational ratios between their locations. Nevertheless, primes are certainly not unions of periodic systems, since they possess a density gradient, and large primes are difficult to predict [2].

In [55], we will use the well-known Dirichlet's theorem on arithmetic progressions to show that the primes in the intervals studied here are limit-periodic in a probabilistic sense. Based on this theorem, we will provide a number-theoretic explanation for the numerical observations here; specifically, the peak location and height formula, the decrease of peak widths, and how the diffuse part vanishes as $L$ increases. Moreover, we will show there that if the interval of the primes is chosen to be appreciably smaller or larger than the one used here, the primes would not be characterized by a large degree of order, as measured by the $\tau$ order metric [40].

The numerical techniques that we employed here to investigate the structure factor of the primes in finite intervals should be applicable to characterize other complex point 


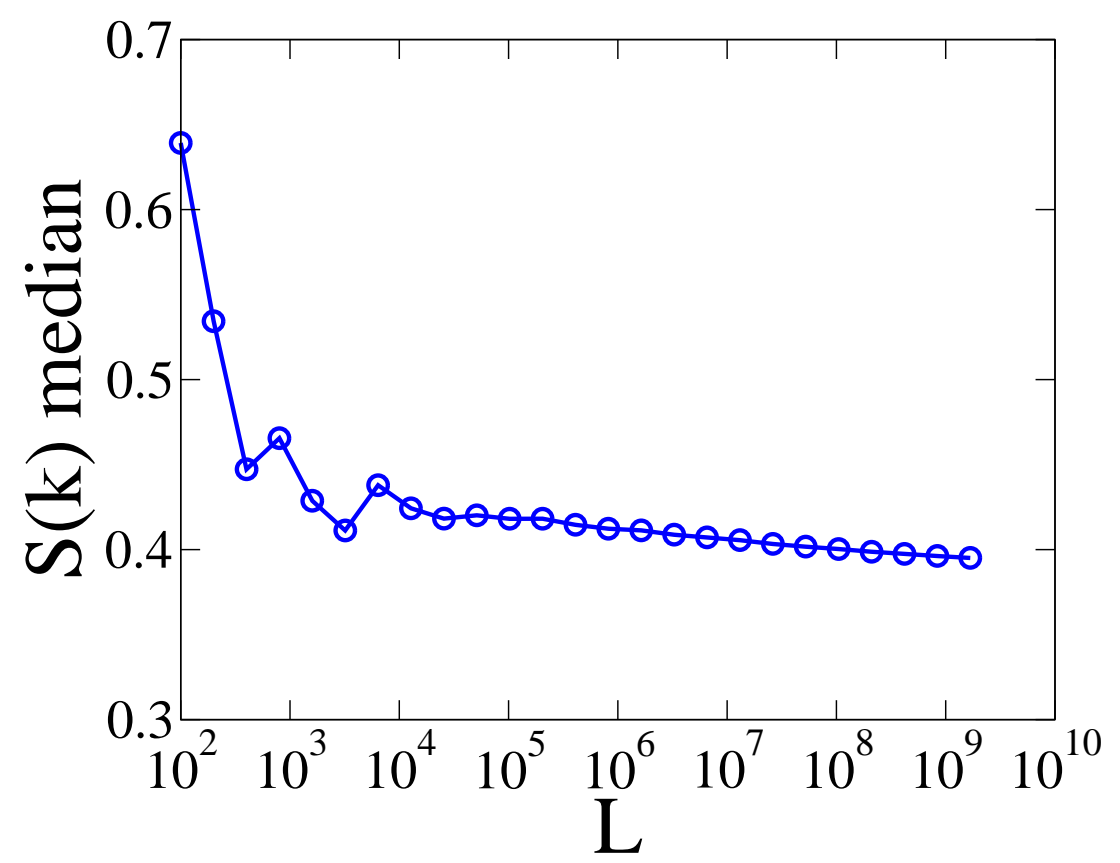

Figure 10. The median of the structure factor, $S(k)$, for all possible choices of $k$, as a function of $L$. Here $M$ is chosen to be $10 L$. The diffuse part of primes appears to be slowly decreasing as $L$ increases. This is to be contrasted with the uncorrelated lattice gas with an appreciably larger predictable diffuse part in which there is no dependence on the system size.

configurations in which the shape and heights of peaks depend sensitively on the system size. These examples include quasicrystals [51] and limit-periodic systems [50]. For example, to study the shape and height of a peak located at $k=2 \pi / \alpha$, the best choice for the system size is a multiple of $\alpha$. Moreover, the structure factor of any finite system with dominant peak structure will always numerically possess a "noisy" diffuse contribution. A simple way to characterize the diffuse part is to calculate the median value of $S(k)$ for all allowed $k$ values and then study its behavior as a function of the system size to see if it becomes negligible in the infinite-size limit.

\section{Acknowledgments}

We are very grateful to Matthew de Courcy-Ireland for helpful discussions. This work was supported in part by the National Science Foundation under Award No. DMR1714722 .

\section{References}

[1] F. R. S. Samuel, "The sieve of eratosthenes. being an account of his method of finding all the prime numbers," Philo. Trans. 62, 327-347 (1772).

[2] https://www.mersenne.org/primes/?press=M74207281.

[3] J. Hadamard, "Sur la distribution des zéros de la fonction $\zeta(s)$ et ses conséquences arithmétiques," Bulletin de la Societé mathematique de France 24, 199-220 (1896). 
[4] A. Granville, "Harald Cramér and the distribution of prime numbers," Scandinavian Actuarial Journal 1995, 12-28 (1995).

[5] P. X. Gallagher, "On the distribution of primes in short intervals," Mathematika 23, 4-9 (1976).

[6] H. L. Montgomery, "The pair correlation of zeros of the zeta function," in Proc. Symp. Pure Math, Vol. 24 (1973) pp. 181-193.

[7] G. Miller, "Riemann's hypothesis and tests for primality," Journal of Computer and System Sciences 13, 300-317 (1976).

[8] M. Rabin, "Probabilistic algorithm for testing primality," J. Number Theory 12, 128-138 (1980).

[9] C. Pomerance, J. L. Selfridge, and S. S. Wagstaff, "The pseudoprimes to $25 \cdot 10^{9}$," Math. Computation 35, 1003-1026 (1980).

[10] R. Baillie and S. S. Wagstaff, "Lucas pseudoprimes," Math. Computation 35, 1391-1417 (1980).

[11] A. O. L. Atkin and F. Morain, "Elliptic curves and primality proving," Math. Computation 61, 29-68 (1993).

[12] A. Granville and G. Martin, "Prime number races," Amer. Math. Monthly 113, 1 (2006).

[13] M. Rubinstein and P. Sarnak, "Chebyshev's bias," Experiment. Math. 3, 173-197 (1994).

[14] F. Martelli, "Dealing with primes i.: On the goldbach conjecture," arXiv:1309.5895 [math.NT], 2013.

[15] Vinogradov I M, The method of trigonometrical sums in the theory of numbers (Dover, Mineola, NY, 2004).

[16] B. Green and T. Tao, "The primes contain arbitrarily long arithmetic progressions," Annals of Math. 167, 481-547 (2008a).

[17] B. Green and T. Tao, "Linear equations in primes," Annals Math. 171, 1753-1850 (2010).

[18] B. Green and T. Tao, "The Mobius function is strongly orthogonal to nilsequences," Ann. Math. 175, 541-566 (2012).

[19] T. Tao and T. Ziegler, "The inverse conjecture for the Gowers norm over finite fields in low characteristic," Annals of Combinatorics 16, 121-188 (2012).

[20] Y. Zhang, "Bounded gaps between primes," Annals of Math. 3, 1121-1174 (2014).

[21] R. J. Lemke Oliver and K. Soundararajan, "Unexpected biases in the distribution of consecutive primes," arXiv:1603.03720 [math.NT] (2016).

[22] J. Maynard, "Small gaps between primes," Annals of Math. 181, 383-413 (2015).

[23] S. R. Dahmen, S. D. Prado, and T. Stuermer-Daitx, "Similarity in the statistics of prime numbers," Physica A 296, 523-528 (2001).

[24] M. Wolfff, "Unexpected regularities in the distribution of prime numbers," Proc. of the 8th Joint EPS - APS Int. Conf. Physics Computing (1996).

[25] P. Kumar, P. C. Ivanov, and H. E. Stanley, "Information entropy and correlations in prime numbers," arXiv:cond-mat/0303110 [cond-mat.stat-mech] (2003).

[26] S. Torquato, A. Scardicchio, and C. E. Zachary, "Point processes in arbitrary dimension from fermionic gases, random matrix theory, and number theory," J. Stat. Mech. Theor. Exp., P11019 (2008).

[27] S. Torquato and F. H. Stillinger, "Local density fluctuations, hyperuniformity, and order metrics," Phys. Rev. E 68, 041113 (2003).

[28] A. Donev, F. H. Stillinger, and S. Torquato, "Unexpected density fluctuations in jammed disordered sphere packings," Phys. Rev. Lett. 95, 090604 (2005).

[29] M. Florescu, S. Torquato, and P. J Steinhardt, "Designer disordered materials with large, complete photonic band gaps," Proc. Natl. Acad. Sci. 106, 20658-20663 (2009).

[30] C. E. Zachary and S. Torquato, "Hyperuniformity in point patterns and two-phase random heterogeneous media," J. Stat. Mech. Theor. Exp. , P12015 (2009).

[31] C. E. Zachary, Y. Jiao, and S. Torquato, "Hyperuniform long-range correlations are a signature of disordered jammed hard-particle packings," Phys. Rev. Lett. 106, 178001 (2011).

[32] R. Kurita and E. R. Weeks, "Incompressibility of polydisperse random-close-packed colloidal particles," Phys. Rev. E 84, 030401 (2011). 
[33] R. Xie, G. G. Long, S. J. Weigand, S. C. Moss, T. Carvalho, S. Roorda, M. Hejna, S. Torquato, and P. J. Steinhardt, "Hyperuniformity in amorphous silicon based on the measurement of the infinite-wavelength limit of the structure factor," Proc. Natl. Acad. Sci. 110, 13250-13254 (2013).

[34] Y. Jiao, T. Lau, H. Hatzikirou, M. Meyer-Hermann, J. C. Corbo, and S. Torquato, "Avian photoreceptor patterns represent a disordered hyperuniform solution to a multiscale packing problem," Phys. Rev. E 89, 022721 (2014).

[35] I. Lesanovsky and J. P. Garrahan, "Out-of-equilibrium structures in strongly interacting Rydberg gases with dissipation," Phys. Rev. A 90, 011603 (2014).

[36] N. Muller, J. Haberko, C. Marichy, and F. Scheffold, "Silicon hyperuniform disordered photonic materials with a pronounced gap in the shortwave infrared," Adv. Opt. Mater. 2, 115-119 (2014).

[37] D. Hexner and D. Levine, "Hyperuniformity of critical absorbing states," Phys. Rev. Lett. 114, 110602 (2015).

[38] R. L Jack, I. R Thompson, and P. Sollich, "Hyperuniformity and phase separation in biased ensembles of trajectories for diffusive systems," Phys. Rev. Lett. 114, 060601 (2015).

[39] C. De Rosa, F. Auriemma, C. Diletto, R. Di Girolamo, A. Malafronte, P. Morvillo, G. Zito, G. Rusciano, G. Pesce, and A. Sasso, "Toward hyperuniform disordered plasmonic nanostructures for reproducible surface-enhanced raman spectroscopy," Phys. Chem. Chem. Phys. 17, 8061-8069 (2015).

[40] S. Torquato, G. Zhang, and F. H. Stillinger, "Ensemble theory for stealthy hyperuniform disordered ground states," Phys. Rev. X 5, 021020 (2015).

[41] ] J. H. Weijs, R. Jeanneret, R. Dreyfus, and D. Bartolo, "Emergent Hyperuniformity in Periodically Driven Emulsions," Phys. Rev. Lett. 115, 108301 (2015).

[42] S. Torquato, "Hyperuniformity and its generalizations," Phys. Rev. E 94, 022122 (2016).

[43] O. Leseur, R. Pierrat, and R. Carminati, "High-density hyperuniform materials can be transparent," Optica 3, 763 (2016).

[44] W.-S. Xu, J. F. Douglas, and K. F. Freed, "Influence of cohesive energy on the thermodynamic properties of a model glass-forming polymer melt," Macromolecules 49, 8341-8354 (2016).

[45] Z. Ma and S. Torquato, "Random scalar fields and hyperuniformity," J. Appl. Phys. 121, 244904 (2017).

[46] H. Davenport and H. L. Montgomery, Multiplicative number theory, Vol. 74 (Springer, New York, 1980).

[47] G. Tenenbaum, Introduction to analytic and probabilistic number theory, Vol. 46 (Cambridge University Press, Cambridge, 1995).

[48] H. Iwaniec and E. Kowalski, Analytic number theory, Vol. 53 (American Mathematical Society, 2004).

[49] M. Baake, R. V. Moody, and P. A. B. Pleasants, "Diffraction from visible lattice points and $k$ th power free integers," Discrete Math. 221, 3 (2012).

[50] M. Baake and U. Grimm, "Diffraction of limit periodic point sets," Phil. Mag. 91, 2661-2670 (2011).

[51] D. Levine and P. J. Steinhardt, "Quasicrystals: a new class of ordered structures," Phys. Rev. Lett. 53, 2477 (1984).

[52] J. E. S. Socolar and P. J. Steinhardt, "Quasicrystals. II.. unit-cell configurations," Phys. Rev. B 34, 617 (1986).

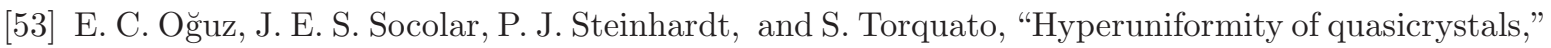
Phys. Rev. B 95, 054119 (2017).

[54] F. Vericat, "A lattice gas of prime numbers and the Riemann Hypothesis," Physica A 392,4516 (2013).

[55] S. Torquato, G. Zhang, and M. de Courcy-Ireland, "Hidden multiscale order in the primes," To be published.

[56] Http://www.primos.mat.br/2T_en.html. 
[57] Https://sourceforge.net/projects/kissfft/. 